\title{
The benefit of botulinum toxin a in the management of central post stroke pain: a case report
}

\begin{abstract}
Purpose: Spasticity and pain are common complications of stroke. Pain following stroke is multi factorial and can be due to musculoskeletal problems, painful spasticity or central post stroke pain. Botulinum Toxin A is the first line pharmacotherapy in spasticity management and increasing evidence has shown benefits in neuropathic pain.

Results: This case report demonstrates the benefit of Botulinum Toxin A in the management of central post-stroke pain with elbow flexor spasticity. The clinical characteristics of the pain were neuropathic and the severity was greater than expected given the degree of spasticity. The pain responded to Botulinum Toxin treatment within a few days, independently of the improvement in spasticity and the effect was continued over six cycles of treatment.

Conclusion: Management of central post stroke pain is challenging due systemic side effects of oral medications. Botulinum Toxin A has increasingly being shown to benefits in neuropathic pain either through blockade of nociceptive substances or acting on central transmission of pain. Although the treatment in our case was given for spasticity management, the patient had unexpected and significant improvement in pain prior to improvement in range of movement. Given the increasing evidence in use of Botulinum Toxin A in neuropathic pain, we feel this may be a possible future treatment option for central post-stroke pain
\end{abstract}

Volume 6 Issue 6 - 2017

\author{
Clare Shippen,' Ganesh Bavikatte,' David \\ Mackarel $^{2}$ \\ 'Speciality Trainee in Rehabilitation Medicine, The Walton \\ Centre NHS Foundation Trust, UK \\ ${ }^{2}$ Honorary Clinical Lecturer Liverpool University, The Walton \\ Centre NHS Foundation Trust, UK
}

Correspondence: Clare Shippen, Speciality Trainee in Rehabilitation Medicine, The Walton Centre NHS Foundation Trust, Liverpool, UK, L9 7LJ, Tel 015 I 556 322I,

Email clareshippen@gmail.com

Received: February 20, 2017| Published: May II, 2017

Keywords: neuropathic pain, spasticity, botulinum toxin, stroke

Abbreviations: CPSP, central post-stroke pain; CRGP, calcitonin gene-related peptide; PSSP, post-stroke shoulder pain

\section{Introduction}

Spasticity is a common problem occurring early after stroke. Many patients with spasticity also have pain. This pain can be musculoskeletal pain, pain provoked by spasticity or spasm, or neuropathic pain. Patients can also experience a mixed picture of pain. Post-stroke neuropathic pain is triggered by pathology in the central nervous system leading to functional abnormalities and is labelled as Central Post-Stroke Pain (CPSP). ${ }^{1}$ These problems have a detrimental effect on quality of life.

Botulinum Toxin $\mathrm{A}$ is the first line pharmacotherapy in the management of spasticity. Following spasticity treatment, pain associated with musculoskeletal problems or painful spasm can improve. Increasing research has also demonstrated the benefit of Botulinum Toxin A in the management of neuropathic pain. ${ }^{2}$ In the case of CPSP, conventional oral treatments are often ineffective due to systemic side effects and pain can be difficult to control. ${ }^{1}$

We present a case of significant improvement in post-stroke neuropathic pain with localised elbow flexor spasticity following the use of Botulinum Toxin A. This has been reported in a report of two cases in which the patients also experienced an unexpected reduction in CPSP post botulinum toxin. However this was improvement lessened with time and analgesia on repeat therapies was not reported. ${ }^{3}$ This case demonstrates a clear long term improvement in a neuropathic pain sensation, which was independent from the improvement in spasticity. The improvement in pain sensation occurred in the first few days following the treatment, before any effect on spasticity. This effect was seen again on six repeat cycles of treatment.

\section{Case study}

This 61 year old lady had a stroke in August 2012 resulting in left sided hemiplegia. She made a good recovery and after 5 days in the acute stroke unit she was discharged home. By January 2013 she started to develop pain in her left arm. This initially started in her left shoulder and then moved to affect her left elbow. She described this pain as a burning and stabbing sensation affecting the left elbow and forearm and was triggered by stress and cold. The pain became more persistent and more severe in nature over time. Functionally, she had difficulty gripping with her left hand resulting in difficulties with dressing and she was unable to drive.

On examination she had no spasticity to her shoulder, wrist or fingers. She had isolated spasticity of her left elbow. She had preserved power apart from mild weakness to left shoulder abduction, triceps and finger extension. She had hyperpathia to the medial aspect of her left elbow. The changes to the left elbow were out of proportion to the clinical findings in the rest of the neurological examination and her pain was more than expected given the degree of spasticity.

She was trialled on gabapentin, pregabalin, amitriptyline, capsaicin cream, lamotrigine, lignocaine patches and tramadol to treat likely central post-stroke pain with no improvement in pain and significant side effects. In March 2015 she had ongoing continuous burning pain described at a constant $9 / 10$ on the visual analogue scale. She received Botox ${ }^{\circledR}$ (OnabotulinumtoxinA) intramuscular injections under ultrasound guidance into left elbow flexors: Biceps Brachii 100 Units, Brachialis 75 Units and Brachioradialis 25 Units (dilution 100units in $2 \mathrm{mls}$ of $0.9 \%$ saline). The goals of treatment were to increase range of movement to her elbow, improve functional tasks such as dressing, and manage pain.

Following the treatment, it took approximately two days for the pain to settle. The spasticity itself did not start to improve until a week 
post injection. In the ongoing weeks she was able to fully straighten her left arm and use this arm for functional tasks. She is now able to dress herself, has gone back to driving and is working on an allotment. She reports significant improvement in mood and social isolation post treatment. The treatment has been repeated at three monthly intervals. She has continued to note pain relief prior to the relief of spasticity. The burning pain then tends to return at ten weeks prior to the return of spasticity. This treatment has been repeated six times at three monthly intervals. The pain relief has been a continued benefit in each injection cycle.

\section{Discussion}

Central post-stroke pain (CPSP) is characterised by pain and sensory abnormalities, which correspondence to the territory of the cerebrovascular lesion. It can be difficult to distinguish between other pain types following a stroke, such as painful spasticity or other musculoskeletal problems. Many patients after stroke can present with multiple pain syndromes and a definite diagnosis of CPSP is difficult. ${ }^{2}$ Allodynia or descriptors such as a burning pain or electric shock sensation can aid the diagnosis. Proposed mechanisms to the development of CPSP include an increase in neuronal hyperexcitability, alterations in spinothalamic tract function or loss of normal inhibition of pain. ${ }^{4}$

The use of Botulinum Toxin in the management of neuropathic pain is increasingly recognized. ${ }^{5}$ In spasticity, Botulinum Toxin is used to reduce muscle hyperactivity by blockade of the pre-synaptic terminal to prevent acetylcholine release. ${ }^{6}$ In pain management, Botulinum Toxin has been shown to inhibit nociceptive neurotransmitters such as substance P, glutamate and Calcitonin Gene-Related Peptide (CRGP). ${ }^{7}$ However evidence has shown that following treatment with Botulinum Toxin for neuropathic pain, there were no changes in total concentrations of substance P or CGRP in biopsy samples. Therefore it was been suggested that the analgesic effect could be related to central mechanisms of pain transmission. ${ }^{8}$

Botulinum toxin has been shown recently to provide pain relief in post stroke spasticity. This study proposed the effect of treatment on spasticity and muscle tone were independent of each other resulting from a direct anti-nociceptive effect from the botulinum toxin. ${ }^{9}$ One report of two cases has demonstrated improvement in central post stroke pain following the use of botulinum toxin. However, in these cases the pain improved following one course of treatment with botulinum toxin. In one case the allodynia has reduced six months after the initial treatment. In the second case, follow up at three months also reported ongoing improvement in pain.

Management of CPSP is challenging as patients tend to respond inadequately to conventional treatments. Antidepressants, anticonvulsants and opiates can be tried. Non-pharmacological treatments include repetitive transcranial magnetic stimulation, deep brain stimulation and motor cortex stimulation. ${ }^{2}$

Among the post-stroke painful conditions, the use of Botulinum Toxin in Post-Stroke Shoulder Pain (PSSP) has been reported to treat pain as well as spasticity. ${ }^{10}$ The pathology of PSSP is unclear. There are theories to suggest the pain is either nociceptive in origin, generated from peripheral pathology, or neuropathic resulting from damage to the spino-thalamo-cortical system. ${ }^{11}$

\section{Conclusion}

This patient had clear evidence of spasticity isolated to her elbow flexors. However this was out of proportion to the pain experienced.
The description was characteristic of neuropathic pain and would be consistent with a diagnosis of CPSP. Despite trials of multiple oral and topical medications, there was limited improvement in pain. The pain significantly improved with the use of Botulinum Toxin, with relief of pain occurring earlier than relief of spasticity. The pain returns in between injection cycles and she has continuing benefit with further treatments. Therefore there was continued improvement in pain with 15 months of repeat treatments. Although we recognise there could be an aspect of reducing spasticity contributing to the analgesic effect found, this does not seem to be the case as the analgesic effect is noted earlier than tone reduction and the neuropathic pain also returns before the return of spasticity.

Increasing evidence suggests that Botulinum Toxin is useful in the management of neuropathic pain and therefore it could be a possible future treatment option for those with CPSP even when not associated with spasticity. More research is required to clarify the potential future role for botulinum toxin in CPSP.

\section{Acknowledgments}

None.

\section{Conflicts of interest}

None.

\section{Funding}

None.

\section{References}

1. Oh H, Seo W A. Comprehensive Review of Central Post-Stroke Pain. Pain Manag Nurs. 2015; 16(5):804818.

2. Brown EA, Schütz SG, Simpson DM. Botulinum Toxin for neuropathic pain and spasticity: an overview. Pain Manag. 2014;4(2):129-151.

3. Camoes-Barbosa A, Neves AF. The Analgesic Effect of Abobotulinum and Incobotulinum Toxins Type A in Central Poststroke Pain: Two Case Reports. PM R. 2016;8(4):384-387.

4. Sim WS. Application of botulinum toxin in pain management. Korean $J$ Pain. 2011;24(1):1-6.

5. Klit H, Finnerup NB, Jensen TS. Central post-stroke pain: clinical characteristics, pathophysiology and management. Lancet Neurol. 2009;8(9):857-868

6. Royal College of Physicians Spasticity in adults: Management using botulinum toxin, National Guidelines. 2009.

7. Patil S, Willett O, Thompkins T, et al. Botulinum Toxin: Pharmacology and Therapeutic Roles in Pain States. Curr Pain Headache 2016 Rep. 2016;20(3):15.

8. Attal N, de Andrade DC2, Adam F3, et al. Safety and efficacy of repeated injections of botulinum toxin $\mathrm{A}$ in peripheral neuropathic pain (BOTNEP): a randomised, double-blind, placebo-controlled trial. Lancet Neurol. 2016;15(6):555-565.

9. Wissel J, Ganapathy V, Ward AB, et al. OnabotulinumtoxinA Improves Pain in Patients With Post-Stroke Spasticity: Findings From a Randomized, Double-Blind, Placebo-Controlled Trial. J Pain Symptom Manage. 2016;52(1):17-26.

10. Singh JA, Fitzgerald PM. Botulinum toxin for shoulder pain. Cochrane Database Syst Rev. 2010; 8(9):CD008271.

11. Intiso D, Basciani M, Santamato A, et al. Botulinum Toxin Type A for the Treatment of Neuropathic Pain in Neuro-Rehabilitation. Toxins (Basel). 2015;7(7):2454-2580. 